\title{
Inhalt, Vol. 4, No. 4, 1981
}

ONKOLOGIE Zeitschrift für Krebsforschung u. -behandlung

unter Fortführung der »österreichischen Zeitschrift für Onkologie«

Band 4, Nummer 4, August 1981

Inhalt

Contents

Impressum 183

Vutuc, Ch. und Kunze, M., Wien

Zigaretten-Rauchkondensat-Exposition und Berufs-

anamnese bei weiblichen Lungenkrebspatienten

Dunzendorfer, U.; Drahovsky, D. und Schmidt-Gayk, H., Frankfurt/Heidelberg

Peptidhormone LH, FSH, TSH, Prolaktin, ß-HCG und und PTH bei Patienten mit urogenitalen

Tumoren .... 188

Hinweise für Autoren 192

Steinke, B.; Benöhr, H. C; Gartner, H. V. und Waller, H. D., Tubingen

Non-Hodgkin-Lymphome mit gastrointestinaler Mani

festation 193

D. Lutz, Wien

Klinik und Immunologie bei akuten myeloischen Leukä-

mien unter einer Chemo-Immunotherapie 202

Sonderbände 212

Pelloni, L.; Wagenknecht, L. und Cavalli, F., Bellinzona

Prognostische Faktoren beim metastasierenden Mela-

nom 213

Kuber, W.; Aiginger, P.; Kühböck, J. und Spona, J., Wien

Wertigkeit der Serum-ß-HCG-Bestimmung bei Patien

ten mit malignen Hodentumoren 221

S. Legha, Houston/Texas

Chemotherapie des Mamma-Karzinoms 226

Onkologie-Mitteilungen 228

Imprint183

Vutuc, Ch. and Kunze, M., Wien

Tobacco Condensate Exposure and Occupational History

in Female Lung Cancer Patients

184

Dunzendorfer, U.; Drahovsky, D., and Schmidt-Gayk, H.,

Frankfurt/Heidelberg

Peptide Hormones in Genito-Urinary Cancer Patients . . 188

Instructions to Authors 192

Steinke, B.; Benöhr, H. C; Gartner, H. V., and 
Waller, H. D., Tubingen

Non-Hodgkin's Lymphomas of the Gastrointestinal Tract 193

D. Lutz, Wien

Clinics and Immunology of Acute Myeloid Leukemias

During Chemo-Immunotherapy 202

Special Editions 212

Pelloni, L., · Wagenknecht, L., and Cavalli, F., Bellinzona

Prognostic Factors in Patients with Advanced Malignant

Melanoma 213

Kuber, W.; Aiginger, P.; Kühböck, J., and Spona, J., Wien

Significance of ß-HCGin Patients with Malignant Tumors

oftheTestis 221

S. Legha, Houston/Texas

Chemotherapy of the Mamma Carcinoma 226

Oncology Communications 228

Beilagen-Hinweis:

Dieser Ausgabe liegt ein Prospektblatt bei von der Hospipharm

Vertriebsgesellschaft mbH, Düsseldorf.

Wir bitten um gfl. Beachtung.

Bibuographischer Hinweis: Inhaltsverzeichmsse dieser Zeitschrift erscheinen regelmäßig in current contents ${ }^{\circledR}$ sowie in anderen bibliographischen Diensten. 